Saudi Journal of Oral and Dental Research

Abbreviated Key Title: Saudi J Oral Dent Res

ISSN 2518-1300 (Print) |ISSN 2518-1297 (Online)

Scholars Middle East Publishers, Dubai, United Arab Emirates

Journal homepage: https://saudijournals.com/sjodr

Review Article

\title{
Chronic Periodontitis as a Factor of Initial and Secondary Systemic Low Intensity
}

\author{
Nataliya Yu. Emelyanova ${ }^{1 *}$, Dmitry V. Emelyanov ${ }^{2}$ \\ ${ }^{1}$ Dentist, Senior Research Associate of the Department of Complex Risk Reduction of Chronic Non-Communicable Diseases, Government Institution \\ "L.T. Malaya Therapy National Institute of the National Academy of Medical Sciences of Ukraine" \\ ${ }^{2}$ Dentist, Associate professor, Department of surgical diseases, operative surgery and topographic anatomy, V. N. Karazin Kharkiv National University, \\ Ukraine
}

DOI: $10.36348 /$ sjodr.2020.v05i07.001

| Received: 23.06.2020 | Accepted: 01.07.2020 | Published: 05.07.2020

*Corresponding author: Nataliya Yu. Emelyanova

\section{Abstract}

The article shows the role of chronic periodontitis in maintaining low-intensity systemic inflammation, which underlies the pathogenesis of many somatic diseases. Some etiological factors of chronic periodontitis, namely the growth of specific microflora, can affect the course of pathological processes or conditions in various organs and systems of the body, which indicates the mandatory examination of the oral cavity in the treatment of any somatic diseases.

Keywords: low-intensity systemic inflammation, chronic periodontitis, periodontopathogenic microflora, somatic diseases.

Copyright @ 2020: This is an open-access article distributed under the terms of the Creative Commons Attribution license which permits unrestricted use, distribution, and reproduction in any medium for non-commercial use (NonCommercial, or CC-BY-NC) provided the original author and source are credited.

\section{INTRODUCTION}

In modern research, when examining the pathogenesis of various somatic (physical) pathologies, the attention of scientists is drawn to the role of lowintensive chronic systemic inflammation, as initiating and maintaining factor in the course of disease. The clinical signs, characteristic of acute inflammation, are not observed in such inflammatory process, and patients usually seek medical advice concerning a specific somatic disease [1].

The anatomical areas are of great scientific and clinical interest due to the fact that there is a high risk of formation of such chronic foci there; they do not bother the patient clinically, but undoubtedly interact with the immune system from the standpoint of initiating and maintaining low-intensive systemic inflammation [2]. The focus on such areas is grounded by the fact that they have constant direct contact with various environmental factors, however, if the balance of this interaction is impaired, the protective potential in these zones decreases and the emergence and course of pathologies with a chronic inflammatory process in the structure of pathogenesis are provoked.

The oral cavity is one of such anatomical areas in the body. The healthy dentofacial sulcus, which is part of the periodontal tissue complex, plays an important role, as the boundary between the stimuli and the internal milieu. The periodontium, in addition to its forming tissues, has a powerful blood supply, which is provided by a dense vascular network. A characteristic dental disease occurs in periodontal tissues under the complex influence of various risk factors, which by the way includes concomitant somatic (physical) pathology; it is called chronic periodontitis (CP) [3-4].

It is a common fact, revealed in scientific studies, that $\mathrm{CP}$ is an ecological niche for the qualitative and quantitative growth of specific periodontalpathogenic microflora, as well as a source of creating pro-inflammatory systemic cytokines, which, penetrating into the capillary vessels, adversely affect the course of somatic diseases in the body [5]. Currently, it has been established that an increase in specific periodontal-pathogenic microflora in the composition of the oral cavity's microbiome is one of $\mathrm{CP}$ etiological factors. These microorganisms are strict anaerobes by the type of respiration; they are found in insignificant amounts in clinically healthy gingiva, however they increase sharply in case of pathology in these tissues [6-7]. Periodontal pathogens have its own destructive potential and immunogenic activity; they are able to penetrate into milieu interieur and provoke the secretion of pro-inflammatory cytokines, supporting low-intensive systemic inflammation. 
It is a common fact that endothelial dysfunction and chronic inflammation are of great importance in the pathogenesis of atherosclerosis. Disruption of microcirculation level, arising under the influence of pathological changes in the vessel wall, will lead to a decrease in the trophism of organs and tissues of any body system with the emergence of a clinical picture of characteristic diseases. According to polymerase chain reaction (PCR), the genetic material of some periodontal pathogens, such as Porphyromonas gingivalis, has been found in atherosclerotic plaques, supporting the inflammatory response in the endothelium and the progression of atherosclerosis [8]. $\mathrm{CP}$ is characterized by the formation of a periodontal pocket instead of a physiological gingival sulcus, in which anaerobic conditions are created and maintained. Such pathological pocket is an ecological niche for the growth and vital functions of periodontal-pathogenic microflora. According to studies, the increased risk of cardiovascular diseases, in the form of myocardial infarction and cardiovascular accident, positively correlates with an increase in the depth of the periodontal pocket during CP progression [9-10].

Patients with diseases of the respiratory system in the form of chronic obstructive pulmonary disease (COPD) should also undergo a dental examination of periodontal tissues. Such somatic (physical) pathology is included in the group of modified CP risk factors, as it creates the conditions for the development and progression of this dental pathology. But, in turn, specific periodontal pathogens, entering the respiratory tract from $\mathrm{CP}$ focus, will be factors, maintaining the inflammatory process in the bronchopulmonary system [11-12].

Long-term results of irrational antibiotic therapy have revealed a significant negative effect of this drugs' group on the adequate functioning of the immune system. It is a common fact that proper programming of the immune system begins immediately after birth, and indigenous microflora mediates and regulates this process; as it begins to actively populate the sections of the gastrointestinal tract (GIT) necessary for its symbiotic relationships with the macroorganism. It is populating, growth and vital activity of the symbiontic microflora in the conditions of physiological biotopes that forms the human microbiome and contributes to the full development and functioning of the immune system [13].

Herewith, negative changes in the microbiome structure can occur not only under the influence of uncontrolled drugs administration, but also due to an increase in the quantitative composition of periodontal pathogens in the oral cavity. Significant changes in the microbiome structure of the oral cavity, in case of $\mathrm{CP}$ presence, are confirmed by studies of the oral fluid, by using PCR. The results of such studies have proved that the incidence of specific periodontal pathogens was statistically higher to compare with patients with healthy dentofacial sulcus (Masato Yoneda and et, 2012) [14]. It has been established that periodontal pathogens, namely $\mathrm{P}$. gingivalis, can cause significant changes in the composition of the entire microflora of the oral cavity, stimulate neutrophil proteinase activity and increase the development of periodontal tissue destruction, as well as have a systemic effect connected with their toxic and immunopathogenetic effects.

Due to the fact that the oral cavity is the initial part of the gastrointestinal tract, and periodontal pathogenic microflora has the ability to get further into the digestive system in general, this is bound to be reflected in the composition of the intestinal microbiota, which actively reacts and shifts in response to various changes in human life (disease, the nature of food, mood, etc.). Recent research data show that specific periodontal pathogens, entering the gastrointestinal tract, can provoke dysbiotic disorders in the intestinal microbiome [15]. Such disturbances contribute to qualitative changes in the composition of the crypt compartment and lead to a decrease in colonization resistance. Disturbance of such an important barrier to the penetration of various pathogens such as colonization resistance contributes to endotoxemia and low-intensive systemic inflammation.

Japanese scientists have conducted an investigation to study changes in the intestinal microbiota in the experiment [16]. It was demonstrated that a single oral administration of $\mathrm{P}$. gingivalis to laboratory rodents provoked changes in the composition of their intestinal microbiota. Changes occurred in the form of an increase of Bacteroidetes percentage and a decrease of Firmicutes percentage, which led to the spread of endotoxin level in blood serum and systemic inflammation.

In the XXI century, according to WHO, the prevalence and intensity of metabolic diseases, leading to early disability and mortality, is growing rapidly, especially in developed countries. Metabolic syndrome (MS) is one of these pathologies; its structure includes several pathologies combined pathogenetically [17]. MS is characterized by obesity, which is reflected by an increase in body mass index, arterial hypertension, insulin resistance, which leads to type II diabetes mellitus (DM), dyslipidemia, and other metabolic diseases. Numerous scientific studies show a decrease in protective potential, the level of microcirculation in periodontal tissues, hypo-salivation in such pathological conditions of the body [18]. All this contributes to CP initiation and, as a result, the formation of a focus of chronic septicemia to maintain the level of lowintensive systemic inflammation. It has also been established that excess adipose tissue is a predisposing factor for low-intensive systemic inflammation and this makes it possible to assume an increase of the negative 
impact on the body of the patients, suffering from obesity and CP.

Risk factors of type II diabetes mellitus include existing foci of chronic inflammation, contributing to the development of insulin resistance and disease progression. On the one hand, diabetes mellitus contributes to the creation of conditions for the occurrence of a chronic inflammatory process in the oral cavity, however $\mathrm{CP}$, in turn, affects the course of this somatic (physical) pathology, supporting the level of low-intensive systemic inflammation [19].

Over the last two decades, nonalcoholic fatty liver disease (NAFLD) is one of the metabolic pathologies that is rapidly gaining worldwide prevalence. According to statistics, about $30 \%$ of people in different countries of the world suffer from various forms of NAFLD and this pathology has a high risk of increasing the prevalence [20-21].

Many studies emphasize the link between CP and NAFLD, especially from the standpoint of a high risk of chronic inflammation in periodontal tissues under the influence of this metabolic pathology. Scientific data from such studies do indicate that the deterioration in the severity of periodontitis is observed with the progression of NAFLD. A group of American scientists has noted that the prevalence of $\mathrm{CP}$ has a positive correlation with the frequency of NAFLD manifestations [22-23]

However, regular penetration of specific periodontal pathogens leads to changes in adipose tissue and liver. Changes of intestinal microbiota cause a decrease of intestinal barrier function due to the release of endotoxins, which provoke systemic inflammation, in particular, in adipose tissue and liver, atherogenic dyslipidemia, which in turn leads to the development of NAFLD [24].

Of course, $\mathrm{CP}$ is a dental disease with a characteristic clinical picture and symptoms; however it requires examination and treatment from the perspective of a holistic organism.

$\mathrm{CP}$ and various somatic diseases interact with each other at the level of aetiopathogenesis, characterized by the ability of adversely affecting each other. Often, somatic (physical) pathology first and foremost becomes a trigger for initiating the inflammatory process in periodontal tissues, creating the necessary conditions for forming a focus of specific periodontal-pathogenic microflora, chronic septicemia, and the secretion of proinflammatory cytokines [25-26].

\section{CONCLUSION}

Low-intensive systemic inflammation is presented in the pathogenesis of numerous somatic diseases; it may be supported by proinflammatory cytokines from a focus, located in another anatomical area. Such a focus as CP, localized allegedly only in the oral cavity, is able to support pathological processes in other organs and body systems due to the dense blood supply [27].

Thus, ignoring a full examination of the oral cavity by patients, seeking medical care at the clinic for internal medicine, will contribute to the failure to obtain the expected and planned treatment results and the possibility of exacerbation or complications of the somatic disease.

\section{REFERENCES}

1. León-Pedroza, J. I., González-Tapia, L. A., del Olmo-Gil, E., Castellanos-Rodríguez, D., Escobedo, G., \& González-Chávez, A. (2015). Low-grade systemic inflammation and the development of metabolic diseases: from the molecular evidence to the clinical practice. Cirugía y Cirujanos (English Edition), 83(6), 543-551.

2. Bennett, J. M., Reeves, G., Billman, G. E., \& Sturmberg, J. P. (2018). Inflammation-Nature's way to efficiently respond to all types of challenges: implications for understanding and managing "the epidemic" of chronic diseases. Frontiers in Medicine, 5, 316.

3. Heart, G. (2020). Global report published on links between periodontal and cardiovascular diseases.

4. Nazir, M.A. (2017). Prevalence of periodontal disease, its association with systemic diseases and prevention. Int J Health Sci (Qassim), 11(2):72-80.

5. Alpert, P.T. (2017). Oral health: the oral-systemic health connection. Home Health Care Manag Pract, 29(1):56-59.

6. Magana, M., Sereti, C., Ioannidis, A., Mitchell, C.A., Ball, A.R., Magiorkinis, E., Chatzipanagiotou, S., Hamblin, M.R., Hadjifrangiskou, M., \& Tegos, G.P. (2018). Options and Limitations in Clinical Investigation of Bacterial Biofilms. Clin. Microbiol. Rev. 31, e00084-16.

7. Kaidonis, J., Townsend,G. (2016). The 'sialomicrobial-dental complex' in oral health and disease. Ann Anat., 203:85-89.

8. Hussain, M., Stover, C. M., \& Dupont, A. (2015). $\mathrm{P}$. gingivalis in periodontal disease and atherosclerosis-scenes of action for antimicrobial peptides and complement. Frontiers in immunology, 6, 45.

9. Saffi, M. A. L., Furtado, M. V., Polanczyk, C. A., Montenegro, M. M., Ribeiro, I. W. J., Kampits, C., ... \& Rabelo-Silva, E. R. (2015). Relationship between vascular endothelium and periodontal disease in atherosclerotic lesions. World journal of cardiology, 7(1), 26.

10. Bourgeois, D., Inquimbert, C., Ottolenghi, L., \& Carrouel, F. (2019). Periodontal Pathogens as Risk Factors of Cardiovascular Diseases, Diabetes, Rheumatoid Arthritis, Cancer, and Chronic 
Obstructive Pulmonary Disease-Is There Cause for Consideration?. Microorganisms, 7(10), 424.

11. Madalli, R., Kheur, S., Reddy, M. G., Kheur, M., \& Mahalle, A. (2016). Assessment of role of Porphyromonas gingivalis as an aggravating factor for chronic obstructive pulmonary disease patients with periodontitis. Dental Hypotheses, 7(3), 100.

12. Rajakaruna, G. A., Negi, M., Uchida, K., Sekine, M., Furukawa, A., Ito, T., ... \& Meinzer, W. (2018). Localization and density of Porphyromonas gingivalis and Tannerella forsythia in gingival and subgingival granulation tissues affected by chronic or aggressive periodontitis. Scientific reports, $8(1)$, $1-13$.

13. Lazar, V., Ditu, L. M., Pircalabioru, G. G., Gheorghe, I., Curutiu, C., Holban, A. M., ... \& Chifiriuc, M. C. (2018). Aspects of gut microbiota and immune system interactions in infectious diseases, immunopathology, and cancer. Frontiers in immunology, 9, 1830.

14. Yoneda, M., Naka, S., Nakano, K., Wada, K., Endo, H., Mawatari, H., ... \& Murata, S. (2012). Involvement of a periodontal pathogen, Porphyromonas gingivalis on the pathogenesis of non-alcoholic fatty liver disease. $B M C$ gastroenterology, 12(1), 16.

15. Kato, T., Yamazaki, K., Nakajima, M., Date, Y., Kikuchi, J., Hase, K., ... \& Yamazaki, K. (2018). Oral administration of Porphyromonas gingivalis alters the gut microbiome and serum metabolome. mSphere, 3(5).

16. Nakajima, M., Arimatsu, K., Kato, T., Matsuda, Y., Minagawa, T., Takahashi, N., ... \& Yamazaki, K. (2015). Oral administration of P. gingivalis induces dysbiosis of gut microbiota and impaired barrier function leading to dissemination of enterobacteria to the liver. PloS one, 10(7), e0134234.

17. Lee, M. K., Han, K., Kim, M. K., Koh, E. S., Kim, E. S., Nam, G. E., \& Kwon, H. S. (2020). Changes in metabolic syndrome and its components and the risk of type 2 diabetes: a nationwide cohort study. Scientific reports, 10(1), 1-8.
18. Des Gachons, C. P., \& Breslin, P. A. (2016). Salivary amylase: digestion and metabolic syndrome. Current diabetes reports, 16(10), 102.

19. Liccardo, D., Cannavo, A., Spagnuolo, G., Ferrara, N., Cittadini, A., Rengo, C., \& Rengo, G. (2019). Periodontal disease: A risk factor for diabetes and cardiovascular disease. International journal of molecular sciences, 20(6), 1414.

20. Ore, A., \& Akinloye, O. A. (2019). Oxidative stress and antioxidant biomarkers in clinical and experimental models of non-alcoholic fatty liver disease. Medicina, 55(2), 26.

21. Cotter, T. G., \& Rinella, M. (2020). NAFLD 2020: The State of the Disease. Gastroenterology.

22. Akinkugbe, A. A., Slade, G. D., Barritt, A. S., Cole, S. R., Offenbacher, S., Petersmann, A., ... \& Heiss, G. (2017). Periodontitis and Non- alcoholic Fatty Liver Disease, a population- based cohort investigation in the Study of Health in Pomerania. Journal of clinical periodontology, 44(11), 1077-1087.

23. Kim, J. Y., Lee, G. N., Song, H. C., Park, Y. M., Ahn, Y. B., Han, K., \& Ko, S. H. (2020). Association between fatty Liver index and periodontitis: the Korea national Health and nutrition examination Survey. Scientific reports, 10(1), 1-7.

24. Shin, H. S. (2020). Association between periodontal status and non- alcoholic fatty liver disease in a Korean adult population: A nationwide cross- sectional study. Journal of Periodontology, 91(4), 524-532.

25. Polak, D., Sanui, T., Nishimura, F., \& Shapira, L. (2020). Diabetes as a risk factor for periodontal disease-plausible mechanisms. Periodontology 2000, 83(1), 46-58.

26. Genco, R. J., \& Borgnakke, W. S. (2020). Diabetes as a potential risk for periodontitis: association studies. Periodontology 2000, 83(1), 40-45.

27. Mawardi, H. H., Elbadawi, L. S., \& Sonis, S. T. (2015). Current understanding of the relationship between periodontal and systemic diseases. Saudi Medical Journal, 36(2), 150. 